\title{
Economía del comportamiento, políticas conductuales y nudges
}

\section{Behavioral economics, behavioral policies and nudges}

\author{
Luis Miller \\ Consejo Superior de Investigaciones Científicas - IPP-CSIC (España) \\ ORCID: https://orcid.org/0000-0002-7447-2313
}

luis.miller@csic.es

\section{NOTA BIOGRÁFICA}

Científico titular del Instituto de Políticas y Bienes Públicos del CSIC. Trabajó como investigador posdoctoral en el Instituto Max Planck de Economía y como adjunto a la dirección e investigador posdoctoral del Centro de Ciencias Sociales Experimentales del Nuffield College de la Universidad de Oxford. Desde 2011 hasta 2018 fue profesor de microeconomía y economía experimental y del comportamiento en la Facultad de Economía y Empresa de la Universidad del País Vasco. Ha realizado estancias de investigación en la Universidad de Essex, la Universidad de Indiana, el Instituto Tecnológico Federal de Zürich, la Universidad Monash de Melburne y la Universidad Carlos III de Madrid. Publica regularmente sobre ciencia social del comportamiento y métodos experimentales en disciplinas como la economía, ciencia política, sociología, psicología y filosofía.

\section{RESUMEN}

Las políticas conductuales, en general, y los nudges, en particular, forman parte de la caja de herramientas que nos permite mejorar el diseño y la aplicación de políticas basadas en la evidencia. Las propuestas de las nuevas ciencias del comportamiento son un complemento de las más tradicionales basadas en incentivos. En este artículo discuto tres ámbitos de aplicación de las políticas conductuales, así como su relación con intervenciones más clásicas, en situaciones que incorporan un nivel de complejidad creciente. Primero, me detendré en situaciones donde nos enfrentamos a problemas de decisión individual. Segundo, en situaciones que tienen en cuenta a otras personas. Por último, abordaré situaciones donde existe interacción social entre personas.

\section{PALABRAS CLAVE}

Políticas conductuales; incentivos; decisiones individuales; preferencias sociales; coordinación.

\begin{abstract}
Behavioral policies, in general, and nudges, in particular, are part of the toolbox that allows us to improve the design and implementation of evidence-based policies. Proposals based on the new behavioral sciences are complementary to traditional incentive-based proposals. In this article, three examples of the application of behavioral policies, as well as their relation to more classic interventions, are discussed in situations incorporating an increasing level of complexity. First, I will focus on situations involving individual decision-making problems. Second, situations in which the decision-maker considers other people. Finally, situations where there is social interaction with other people.
\end{abstract}

\section{KEYWORDS}

Behavioral policies; incentives; individual decisions; social preferences; coordination. 
GAPP. Nueva Época - N. 25, marzo 2021 - ISSN: 1989-8991 - DOI: https://doi.org/10.24965/gapp.i25.10866 - [Págs. 46-57] Número monográfico - Los nudges y el diseño conductual de políticas públicas

Economía del comportamiento, políticas conductuales y nudges

Luis Miller

\begin{abstract}
SUMARIO
1. INTRODUCCIÓN. 2. PREFERENCIAS, INCONSISTENCIAS E INTERVENCIONES CONDUCTUALES. 3. POLITICAS CONDUCTUALES Y DECISIONES INDIVIDUALES. 3.1. DECISIONES INTERTEMPORALES Y DEBILIDAD DE LA VOLUNTAD. 3.2. DECISIONES BAJO RIESGO E INCERTIDUMBRE. 4. POLÍTICAS CONDUCTUALES EN UN MUNDO PARCIALMENTE COOPERATIVO. 5. POLITICAS CONDUCTUALES E INTERACCIÓN SOCIAL. 6. CONCLUSIONES. REFERENCIAS BIBLIOGRÁFICAS.
\end{abstract}

\title{
1. INTRODUCCIÓN
}

El éxito de la aproximación conductual a las políticas públicas en los últimos años es innegable. Más de doscientas organizaciones públicas y privadas en todo el mundo ${ }^{1}$ aplican en la actualidad teorías conductuales al diseño de políticas e intervenciones en cuestiones como la mejora de los hábitos saludables o el incremento del ahorro para el futuro. Pero, ¿y si este éxito escondiera al mismo tiempo una de las mayores amenazas para las políticas conductuales? Me refiero al peligro de aplicar el recetario proporcionado en los libros de divulgación sin haber aprendido suficientemente acerca de los fundamentos que se hallan detrás de esta aproximación científica y de cómo la misma no es la panacea (como ninguna lo es), sino más bien otra aportación más a la caja de herramientas teórica que nos permite mejorar el diseño y la aplicación de políticas basada en la evidencia ${ }^{2}$.

La tesis central que planteo en este artículo es que las propuestas de las nuevas ciencias del comportamiento son más bien complementarias a las propuestas más tradicionales basadas en incentivos. En este sentido, mi objetivo principal es revisar una serie de grandes temas de la economía del comportamiento relacionados con el diseño de políticas e intervenciones, mostrando cómo cada uno de ellos plantea preguntas que pueden ser respondidas tanto a partir de aproximaciones más clásicas basadas en incentivos, como de propuestas más novedosas basadas en modelos conductuales. El uso de una aproximación u otra dependerá, más bien, de la definición del problema, de otros factores contextuales o, en muchas ocasiones, de la factible que sea poner en marcha intervenciones de un tipo u otro.

El repaso que realizo se estructura en torno a tres tipos de situaciones que incorporan un nivel de complejidad creciente. Primero, me detengo en situaciones donde nos enfrentamos a problemas de decisión individual que suelen regularse públicamente, como los juegos de azar o el consumo de alcohol. En este tipo de situaciones, el Estado interviene haciendo más costoso un comportamiento que puede ser dañino para la persona, sobre todo a largo plazo. En segundo lugar, repasaré situaciones donde al tomar decisiones tenemos en cuenta a otras personas, como cuando realizamos una donación a una entidad benéfica. Aquí el Estado u otras organizaciones suelen funcionar más como impulsores o catalizadores de la propensión humana a la cooperación y el comportamiento prosocial. Por último, abordaré situaciones donde existe interacción social o estratégica entre personas, donde el Estado suele intervenir para solucionar problemas de coordinación social, como en el caso de las emergencias sanitarias y las catástrofes naturales.

Pero antes de centrarme en cada uno de esos bloques, le dedicaré unas breves líneas a describir cuáles son esas limitaciones que la nueva economía del comportamiento ha detectado en la economía estándar o neoclásica y que hacen que esta última nos ofrezca solo una visión parcial de la realidad.

\section{PREFERENCIAS, INCONSISTENCIAS E INTERVENCIONES CONDUCTUALES}

Si uno revisa cualquier libro de microeconomía, encontrará en los primeros capítulos una descripción de los supuestos fundamentales de la teoría económica de la decisión. Se trata de propiedades matemáticas que permiten sostener el elemento clave de esta teoría: la consistencia. Por consistencia se entiende que cuando una persona se enfrenta al mismo problema de decisión varias veces, las sucesivas decisiones que

1 https://www.oecd.org/gov/regulatory-policy/behavioural-insights.htm.

2 El peligro al que me refiero no es nuevo. Para una discusión del mismo en el contexto de la Gran Recesión que comenzó en 2008, conviene recordar la contraposición entre la recepción entusiasta de la «revolución conductual» que realizaba el columnista David Brooks en 2008 y la respuesta más moderada de los científicos sociales George Loewenstein y Peter Ubel dos años más tarde. Para más detalles, ver https://www.nytimes.com/2008/10/28/opinion/28brooks.html y https://www.nytimes.com/2010/07/15/ opinion/15loewenstein.html. 
GAPP. Nueva Época - N.o 25, marzo 2021 - ISSN: 1989-8991 - DOI: https://doi.org/10.24965/gapp.i25.10866 - [Págs. 46-57] Número monográfico - Los nudges y el diseño conductual de políticas públicas

Economía del comportamiento, políticas conductuales y nudges

Luis Miller

toma van en la misma dirección ${ }^{3}$. En otras palabras, las decisiones se ajustan a la estructura de incentivos de una situación dada, pero las preferencias de una persona están bien definidas, no se modifican de una situación a otra ${ }^{4}$. Esta consistencia en la toma de decisiones, sin embargo, no predice muchas acciones de un ser humano que tiende a cambiar de opiniones o preferencias dependiendo del contexto. De ahí que los distintos compendios sobre la economía del comportamiento se centren justamente en describir las múltiples inconsistencias humanas y los nudges se planteen en muchas ocasiones como soluciones a estas inconsistencias ${ }^{5}$.

Como veremos en la siguiente sección, mucha de la literatura sobre nudges propone medidas para contrarrestar dos tipos de estas inconsistencias. En primer lugar, la inconsistencia temporal que nos hace tomar decisiones distintas dependiendo de si la decisión afecta al momento presente o al futuro. Mi ejemplo preferido es que siempre estoy dispuesto a salir a correr a la mañana siguiente, pero cuando llega esa mañana raramente lo hago ${ }^{6}$. En segundo lugar, la inconsistencia resultante de cambiar mi elección dependiendo del contexto de decisión. Un ejemplo clásico son las opciones por defecto. Pensemos en todas las opciones que aceptamos cuando instalamos una nueva aplicación tecnológica en nuestro teléfono independientemente de si estas se ajustan más a lo que queremos. Una vez que definimos una elección como la opción por defecto, tendemos a elegirla con más frecuencia. Esto puede tener consecuencias de enorme importancia para la sociedad, como que el mero hecho de hacer que las personas sean donantes por defecto incrementa sustancialmente el número de donaciones (Johnson y Goldstein, 2013).

Una vez que décadas de investigación conductual han mostrado numerosas inconsistencias en la toma de decisiones humanas, la cuestión es qué teoría o conjunto de teorías nos pueden guiar en la formulación de políticas públicas conductuales. Esta pregunta ha recibido respuestas diferentes por parte de la economía neoclásica y de la psicología y economía del comportamiento. La respuesta económica en muchos casos ha sido: «si los humanos no se comportan como prescribe la teoría de la decisión, no podemos concluir que sean irracionales. De hecho, podrían simplemente ser ignorantes o estar mal informados». Es lo que se conoce como «error de actuación» (performance error), es decir, no es que la teoría prediga mal el comportamiento humano, sino que esta necesita realizar supuestos distintos sobre el mismo, asumiendo que los humanos somos limitados y cometemos errores más o menos sistemáticos (Gintis, 2009). Por el contrario, muchos psicólogos y economistas del comportamiento no asumen que los humanos cometan errores (sean inconsistentes) continuamente, sino que, al tomar decisiones, estos «utilizan un número limitado de principios heurísticos que reducen tareas complejas como asignar probabilidades o predecir eventos a operaciones más simples». Esta última aproximación es la que, a través de los denominados heurísticos y sesgos, sirve como base a la idea del nudge (Gilovich et al., 2002).

Para visualizar las distintas aproximaciones teóricas que subyacen al uso de políticas públicas conductuales, hay dos metáforas que describen a la perfección las aproximaciones neoclásica o estándar y conductual. La primera es el denominado «taller de reparaciones neoclásico» (neoclassical repair shop) propuesto por Brandstätter y Güth (1994). En este taller de reparaciones los economistas neoclásicos, tras observar un fenómeno que se escapa a la teoría recibida, tratan de racionalizar ese comportamiento observado modificando de forma ad hoc sus modelos formales. La segunda metáfora, más cercana a los fundamentos teóricos de las políticas públicas conductuales, es la metáfora de las tijeras propuesta por Simon (1955), que enfatiza que las decisiones humanas están limitadas por dos fenómenos (como las dos hojas de unas tijeras): la información disponible y las limitaciones cognitivas, por una parte, y la cantidad finita de tiempo que una persona tiene para tomar una decisión, por otra.

Por tanto, las políticas conductuales parten del reconocimiento de las limitaciones e inconsistencias de la teoría económica estándar basada en incentivos y de la necesidad de diseñar políticas fundamentadas en una concepción más amplia del ser humano. Esta concepción más amplia suele partir de dos conceptos psicológicos fundamentales: los sesgos y los heurísticos.

3 Para un tratamiento del problema de la consistencia en la toma de decisiones y su relación con otros supuestos de la teoría económica estándar, ver Gintis (2009).

4 Es el famoso de gustibus non est disputandum de Stigler y Becker (1976), es decir, que las preferencias (los gustos) de las personas no cambian.

5 Thaler y Sustein (2008, p. 6) definen nudge como «Cualquier aspecto de la arquitectura de elección (choice arquitecture) que altera el comportamiento de las personas de una manera predecible sin prohibir ninguna opción o cambiar significativamente sus incentivos económicos». Por tanto, en la propia definición del término se plantea una contraposición con las intervenciones basadas en incentivos económicos.

6 La literatura sobre cómo la arquitectura de elección nos puede ayudar a promocionar los hábitos saludables en nuestra vida cotidiana, en general, y los relacionados con el ejercicio físico en particular, es aún escasa. Para una revisión reciente, ver Forberger et al. (2019) 
GAPP. Nueva Época - N. ${ }^{\circ}$ 25, marzo 2021 - ISSN: 1989-8991 - DOI: https://doi.org/10.24965/gapp.i25.10866 - [Págs. 46-57] Número monográfico - Los nudges y el diseño conductual de políticas públicas

Economía del comportamiento, políticas conductuales y nudges

Luis Miller

Cuando hablamos de sesgos generalmente hablamos de desviaciones de una determinada teoría normativa, en este caso la teoría económica. Es decir, solo son sesgos en cuanto que los comparamos con esa teoría. En este sentido, la mayoría de sesgos tienen que ver con inconsistencias en la toma de decisiones. Por ejemplo, el «sesgo del presente» (O'Donoghue y Rabin, 2015) hace referencia a las diferencias entre las decisiones que tomamos en el presente (no salgo a correr) y las que tomamos en el futuro, incluso cuando el futuro es dentro de una hora (en una hora seguro que salgo).

Los heurísticos son reglas de decisión simples y eficientes que las personas usan para formarse sus propios juicios o tomar decisiones. Un ejemplo de esto es el heurístico de disponibilidad mediante el cual tendemos a estimar la frecuencia de una clase de eventos o la probabilidad de que un evento pase por la facilidad con las que nos vienen a la cabeza ejemplos de ese evento o clase de eventos. Por ejemplo, tendemos a estimar la probabilidad de morir en un accidente de tráfico basándonos en las veces que recordamos haberlo visto en las noticias, con lo que tendemos a sobreestimar esa probabilidad (Tversky y Kahneman, 1974).

Por último, a pesar de un repertorio teórico y conceptual creciente, las intervenciones conductuales y los nudges no se basan en una teoría de la decisión unificada, como ocurría en el modelo estándar, sino en un conjunto de teorías parciales. Si bien estas teóricas suelen tener su fundamento en la idea de racionalidad limitada o acotada (bounded rationality) de Herbert Simon (Gigerenzer y Selten, 2002), no existe una única teoría de referencia. Uno de los intentos más elaborados de unificación de este conjunto de teorías es el «sistema dual» desarrollado por Kahneman (2012), donde propone que el pensamiento y la toma de decisiones humanas puede ser descrito a través de dos sistemas: el «sistema 1» se encargaría de las decisiones rápidas, automáticas y emocionales y el «sistema 2» de las decisiones lógicas y conscientes ${ }^{7}$.

\section{POLÍTICAS CONDUCTUALES Y DECISIONES INDIVIDUALES}

Muchos de los ejemplos descritos en los textos sobre políticas conductuales atañen a problemas de decisión individual. Ejemplos como el poco nivel de ahorro a largo plazo de las familias, el poco ejercicio físico o el alto consumo de alcohol y drogas son problemas que, aunque tienen claras ramificaciones sociales, en principio tienen que ver con comportamientos individuales. Cuando analizamos los sesgos que afectan al comportamiento individual, dos conjuntos de situaciones destacan sobre el resto: situaciones que requieren de decisiones intertemporales, donde la decisión está distanciada temporalmente de sus consecuencias, y situaciones bajo riesgo o incertidumbre, en las que no somos capaces de calcular la probabilidad asociada a las consecuencias de nuestros actos. En esta sección describiré cuáles son los problemas comunes relacionados con estos dos conjuntos de situaciones, así como las políticas que se han diseñado para combatirlos, tanto desde la óptica tradicional de los incentivos, como desde los enfoques conductuales que implican algún tipo de nudge.

\subsection{Decisiones intertemporales y debilidad de la voluntad}

El tipo de problema estrella que tratan de resolver un sinfín de políticas conductuales es lo que denominamos debilidad de la voluntad. Cuando existe un largo período de tiempo entre la decisión que tomamos y los beneficios o costes provocados por esta, las personas tendemos a tomar decisiones sensatas, pero cuando los costes o los beneficios son inmediatos, las personas tomamos malas decisiones, sacrificando las ganancias a largo plazo por las ganancias inmediatas. Entre los ejemplos más destacados tenemos multitud de casos de comportamiento impulsivo, como el consumo de tabaco y drogas, el sexo no seguro, comer en exceso o el abandono escolar. Otro problema relacionado con la debilidad de la voluntad es el procrastinar. Tanto psicólogos como economistas del comportamiento han mostrado una y otra vez en el laboratorio que las personas tendemos a sacrificar beneficios a largo plazo (por ejemplo, tener una forma física envidiable) por satisfacciones inmediatas (un trozo de tarta). Esto plantea un problema a la teoría de la decisión clásica que suponía que las personas descontamos el futuro de forma constante, es decir, que somos capaces de anticipar y tener en cuenta hoy los beneficios que nos aportará una acción en cualquier momento del futuro y también de comparar esos beneficios (y costes) en dos momentos concretos del tiempo. En realidad, ningún humano funciona de esa forma.

\footnotetext{
7 Sobre la relación de la propuesta de Kahneman con los nudges, ver el artículo de Pedro Rey en este monográfico.
} 
GAPP. Nueva Época - N. 25, marzo 2021 - ISSN: 1989-8991 - DOI: https://doi.org/10.24965/gapp.i25.10866 - [Págs. 46-57] Número monográfico - Los nudges y el diseño conductual de políticas públicas

Economía del comportamiento, políticas conductuales y nudges Luis Miller

Pero, ¿si no descontamos el futuro de forma constante, cómo lo hacemos? Pues aquí es donde, como decía anteriormente, se abren dos conjuntos de teorías: aquellas que no renuncian a proponer un modelo general que pueda explicar la mayoría de comportamientos, es decir, teorías recién salidas del taller de reparaciones neoclásica, y aproximaciones conductuales basadas en sesgos y heurísticos. La más importante entre las primeras es el denominado «modelo de descuento hiperbólico». Me detendré un momento en ese modelo dada su importancia, para centrarme después en aproximaciones conductuales más recientes.

La idea detrás del modelo de descuento hiperbólico (Laibson, 1997) es tremendamente simple: cuando los humanos tomamos decisiones distinguimos entre el presente y cualquier punto en el futuro, independientemente de lo cerca que se encuentre este en el tiempo. Cuando consideramos acciones en el futuro (hacer una dieta o estudiar para el próximo examen), somos consistentes y descontamos cada período de tiempo de forma similar, es decir, adelantar el esfuerzo del momento cuatro al tres es evaluado de igual modo que adelantarlo del momento tres al dos. Sin embargo, el presente es completamente distinto a cualquier otro momento y el valor (coste en este caso) de la acción que vamos a realizar aumenta drásticamente cuando nos movemos del período dos al momento presente. El hecho de que el valor de la acción cambie drásticamente una vez que no estamos en el presente y que después se mantenga constante en el resto de períodos, hace que la curva que describe este valor se asemeje a una hipérbole, de ahí el nombre. En el modelo exponencial típicamente considerado en la economía estándar, no existe esa distinción entre el presente y el resto de puntos temporales, preferimos obtener una recompensan más pronto que tarde o realizar una acción costosa más tarde que pronto, pero no existe esa distinción drástica con el presente.

El modelo de descuento hiperbólico no deja de ser una abstracción o simplificación de la realidad. Sin embargo, sabemos gracias a cientos de experimentos que describir las decisiones intertemporales es algo mucho más complejo. Aún así puede ayudarnos a entender algunos fenómenos importantes que han sido objeto de políticas conductuales, como el bajo nivel de ahorro de las familias. Sin embargo, existen muchos otros fenómenos temporales interesantes desde el punto de vista de las políticas conductuales que no pueden ser captados por el descuento hiperbólico. La Tabla 1 presenta algunos de estos fenómenos descritos por Frederick y otros (2002).

TABla 1. Modelos alternativos de PREFERENCiAs INTERTEMPorales

\begin{tabular}{ll}
\hline \multicolumn{1}{c}{ Modelo alternativo } & \multicolumn{1}{c}{ Descripción } \\
\hline Formación de hábitos & $\begin{array}{l}\text { Las decisiones en el presente (p. e., de consumo) pueden verse afectadas por las que } \\
\text { tomamos en el pasado. }\end{array}$ \\
\hline Puntos de referencia & $\begin{array}{l}\text { Los resultados son evaluados teniendo como referencia un determinado estado o punto } \\
\text { de referencia e interpretamos cualquier resultado como una desviación de este punto. }\end{array}$ \\
\hline Utilidad de la anticipación & La anticipación de un evento futuro puede provocarnos utilidad. \\
\hline Influencias viscerales & $\begin{array}{l}\text { Nuestras decisiones se ven afectadas por cuestiones como el hambre, el deseo se- } \\
\text { xual, el dolor físico o las ansias. }\end{array}$ \\
\hline Sesgo de proyección & $\begin{array}{l}\text { Aunque podemos anticipar cómo van a cambiar cualitativamente nuestras preferencias } \\
\text { en el futuro, tendemos a infraestimar la magnitud de estos cambios. }\end{array}$ \\
\hline Cuentas mentales & $\begin{array}{l}\text { No tratamos todo el dinero u otros bienes del mismo modo, sino que asignamos distin- } \\
\text { tos tipos de gasto a distintas «cuentas mentales». }\end{array}$ \\
\hline $\begin{array}{l}\text { Decisiones entre paréntesis } \\
\text { (choice bracketing) }\end{array}$ & $\begin{array}{l}\text { Una persona solo toma un número reducido de decisiones al mismo tiempo y general- } \\
\text { mente ignora la relación de estas decisiones con otras tomadas en el pasado o que se } \\
\text { tomarán en el futuro. }\end{array}$ \\
\hline Yo múltiple & $\begin{array}{l}\text { El ser humano consistiría en un conjunto de «yoes», unos que se encargan de las de- } \\
\text { cisiones a corto plazo y otros de las decisiones a largo plazo. Este modelo plantea el } \\
\text { problema de la cooperación entre estos múltiples «yoes». }\end{array}$ \\
\hline Utilidad de la tentación & \begin{tabular}{l} 
Uno puede experimentar desutilidad por no elegir la opción más placentera ahora. \\
\hline
\end{tabular}
\end{tabular}

Fuente: Elaboración propia a partir de Frederick y otros (2002). 
GAPP. Nueva Época - N.o 25, marzo 2021 - ISSN: 1989-8991 - DOI: https://doi.org/10.24965/gapp.i25.10866 - [Págs. 46-57] Número monográfico - Los nudges y el diseño conductual de políticas públicas

Economía del comportamiento, políticas conductuales y nudges

Luis Miller

Como he mostrado en la Tabla 1, los problemas relacionados con la toma de decisiones intertemporales son múltiples y complejos. Pero, ¿qué soluciones ofrece la literatura científica para los mismos? Muchas de las soluciones conductuales pasan por adquirir un compromiso en el presente que nos obligue a llevar a cabo aquello que queremos hacer en el futuro. Un ejemplo clásico son las «fechas límite» (deadline) que nos comprometen en el presente a realizar una acción en el futuro (Ariely y Zakay 2001). Otros ejemplos están relacionados con superar los impulsos iniciales a realizar acciones contraproducentes, por ejemplo, en el supermercado dejo de comprar comida a la que no voy a ser capaz de resistirme en casa (Galla y Duckworth, 2015). Este último ejemplo muestra muy a las claras nuestras inconsistencias temporales. Cuando estoy en el supermercado tomo decisiones sobre acciones que realizaré en el futuro, cuando llegue a casa, y, de ese modo, puedo evitar las malas decisiones fruto de la impulsividad. Pero, jcuidado!, dado que también disfrutamos con la anticipación, tampoco estamos completamente a salvo en el supermercado. Un último ejemplo muy interesante que cuenta Kucharski (2020) es que en el Reino Unido se consiguió disminuir drásticamente la tasa de suicidios mediante el consumo de paracetamol cambiando el modo en el que este era proporcionado, de líquido a tabletas. El mero coste de tener que extraer todas las tabletas parece que disuadía a un buen número de las personas que estaban pensando en cometer un suicidio.

Pero no todo son soluciones conductuales. La teoría clásica de los incentivos también ha aportado su granito de arena a superar la debilidad de la voluntad y la impulsividad. Charness and Gneezy (2009) realizaron un experimento de campo en el que pagaban a las personas por ir al gimnasio varias veces al mes y encontraron que no solo incrementaban la actividad directamente incentivada, sino que algunas personas seguían yendo al gimnasio una vez retirados los incentivos materiales, lo que en conjunto mejoraba algunos indicadores de salud de los participantes, como el peso o el ritmo cardiaco ${ }^{8}$.

\subsection{Decisiones bajo riesgo e incertidumbre}

El segundo gran conjunto de decisiones individuales donde los humanos solemos mostrarnos inconsistentes son las decisiones que implican algún tipo de riesgo e incertidumbre. Los meses que siguieron a la llegada de la pandemia de la COVID-19 en 2020 en todo el mundo estuvieron llenos de este tipo de decisiones a todos los niveles, tanto por parte de los gobiernos, como de otras organizaciones como las empresas y los colegios, o de personas individuales. Y en muchos casos se ha recurrido a las ciencias conductuales y a los nudge para manejarnos en situaciones en las que no están claras las consecuencias de nuestros actos (mantener la distancia interpersonal en nuestras interacciones, por ejemplo) o cuál es la probabilidad de que se den estas consecuencias (un contagio, por ejemplo). Pero para entender bien este tipo de situaciones y cómo las políticas conductuales nos pueden ayudar con ellas, debemos entender primero qué son las decisiones bajo riesgo e incertidumbre y por qué nos suponen un problema.

Aunque en el lenguaje cotidiano muchas veces utilizamos de forma indistinta los conceptos de riesgo e incertidumbre, estos son utilizados por la investigación conductual de forma diferente y precisa siguiendo la definición clásica de Knight (1921). Según este economista norteamericano, las situaciones donde existe riesgo son aquellas en las que podemos calcular la probabilidad con que se va a dar un evento concreto. Un ejemplo clásico es una lotería o juego de azar. Por ejemplo, cuando lanzo un dado muchas veces sé que, en media, la probabilidad de que salga un seis es un sexto. Por el contrario, la incertidumbre implica situaciones donde no puedo calcular estas probabilidades. Por ejemplo, dada la falta de información científica sobre las probabilidades de contagio de la COVID-19, tanto instituciones como individuos toman decisiones bajo incertidumbre en respuesta la misma. La distinción entre riesgo e incertidumbre es importante para las políticas conductuales porque mientras que sabemos mucho acerca de cómo tomamos decisiones bajo riesgo, la investigación acerca de las decisiones cuando hay incertidumbre es mucho más escasa.

La investigación acerca de las decisiones bajo riesgo ha dado lugar a la principal teoría alternativa a la teoría económica estándar: la teoría prospectiva o prospect theory, desarrollada por los psicólogos Tversky and Kahneman (1981). Esta teoría parte del supuesto de que debemos distinguir dos fases en la toma de decisiones humana: una fase inicial en la que nuestros actos y sus consecuencias son «enmarcadas» y una fase siguiente donde las acciones y sus consecuencias son evaluadas con respecto al marco utilizado. La idea de «marco» se refiere a un punto de referencia neutral a partir del cual definimos los resultados de

8 Para una revisión de en qué circunstancias pueden servir los incentivos materiales para cambiar el comportamiento, ver Gneezy et al. (2011) 
GAPP. Nueva Época - N. 25, marzo 2021 - ISSN: 1989-8991 - DOI: https://doi.org/10.24965/gapp.i25.10866 - [Págs. 46-57] Número monográfico - Los nudges y el diseño conductual de políticas públicas

Economía del comportamiento, políticas conductuales y nudges

Luis Miller

nuestras acciones como desviaciones negativas o positivas del mismo. Por ejemplo, si recibimos un bonus en el trabajo no lo evaluamos en el vacío, sino en relación con un punto de referencia que podría ser un bonus anterior. Por tanto, el mismo pago podría ser enmarcado de forma positiva por una persona si está por encima del bonus anterior o de forma negativa por otra si supone una bajada (Hossain y List, 2012). Incluso la misma persona podría percibir un bonus de forma diferente en momentos distintos de su carrera teniendo en cuenta los puntos de referencia en cada momento. La investigación también ha mostrado que nuestra reacción ante desviaciones negativas del punto de referencia (cuando perdemos) son más extremas que nuestra reacción a desviaciones positivas (cuando ganamos). Esto tiene como consecuencia que la mayoría de personas seamos renuentes al riesgo y evitamos situaciones que nos puedan suponer una pérdida, aunque también una ganancia similar.

Camerer (2000) describe un buen número de situaciones cotidianas donde se da este efecto de aversión al riesgo. Por ejemplo, el denominado «efecto disposición» en el comportamiento financiero, por el que los inversores venden demasiado rápido activos que están subiendo de valor y mantienen demasiado tiempo activos que van acumulando pérdidas. En realidad, esto no es solo un efecto que se dé en las finanzas, sino también en situaciones cotidianas como la venta de objetos de segunda mano, incluida la venta de una vivienda. También es fácil pensar que los gobiernos siguen manteniendo políticas, aunque las pérdidas se acumulen, para no reconocer los efectos negativos.

La teoría prospectiva tiene una segunda predicción muy importante para las políticas conductuales y es que las personas solemos tener una concepción distorsionada de las probabilidades, sobrevalorando eventos que tienen una probabilidad de ocurrencia muy baja (como un accidente de avión) e infravalorando eventos con probabilidades moderadas o altas (como tener colesterol). Eso hace que, como mostraran Hertwig et al. (2005), nuestra percepción acerca de las causas de muerte esté distorsionada asignando una probabilidad subjetiva demasiado alta a morir de causas que en realidad son poco probables, como los asesinatos o los accidentes, y asignar una probabilidad menor a las causas más comunes como el cáncer o las enfermedades en general. En este caso, las políticas conductuales más clásicas, como las políticas informativas, pueden ser muy efectivas ya que se trata de cambiar una distorsión cognitiva bastante común. Otras distorsiones pueden ser menos fáciles de combatir. La Tabla 2 muestra algunas de las consecuencias mejor documentadas de la teoría prospectiva.

TABLA 2. Distorsiones COMUNES DERIVADAS DE LA TEORÍA PROSPECTIVA

\begin{tabular}{ll}
\hline \multicolumn{1}{c}{ Distorsión } & \multicolumn{1}{c}{ Descripción } \\
\hline Cuenta psicológica & $\begin{array}{l}\text { Se trata de un marco de resultados que especifica: (i) un conjunto de resultados elemen- } \\
\text { tales que son evaluados de forma conjunta y de qué manera son combinados y (ii) un } \\
\text { resultado de referencia que es considerado neutral o normal. }\end{array}$ \\
\hline Cuenta mínima & $\begin{array}{l}\text { Las personas generalmente solo evalúan los actos en relación con las consecuencias } \\
\text { directas de estos y no con consecuencias indirectas que estos puedan tener. }\end{array}$ \\
\hline Efecto de coste hundido & $\begin{array}{l}\text { Se da cuando una decisión hace referencia a una cuenta mental que se encuentra en } \\
\text { negativo. }\end{array}$ \\
\hline Efecto dotación & Las personas a menudo demandan mucho más para desprenderse de un objeto de lo que \\
(endowment effect) & estarían dispuestas a pagar para adquirirlo. \\
\hline Efecto de statu quo & $\begin{array}{l}\text { Las personas tienen una fuerte tendencia a permanecer en el statu quo porque las incon- } \\
\text { veniencias de abandonarlo se prevén mayores que las ventajas de hacerlo. }\end{array}$ \\
\hline
\end{tabular}

Fuente: Elaboración propia a partir de Kahneman et al. (1991).

De nuevo, algunos ejemplos relacionados con la reciente pandemia de la COVID-19 pueden ayudarnos a entender la importancia social y para las políticas públicas de entender adecuadamente las decisiones bajo riesgo. Así, en el diseño de la denominada «desescalada» o conjunto de medidas de apertura tomadas en España después del confinamiento generalizado en la primavera de 2020, se discutió sobre si era más adecuado introducir medidas de forma secuencial, en distintas fases, o hacerlo de forma generalizada. El problema de hacerlo de forma secuencial era que planteaba un escenario donde el statu quo se iba cam- 
GAPP. Nueva Época - N.o 25, marzo 2021 - ISSN: 1989-8991 - DOI: https://doi.org/10.24965/gapp.i25.10866 - [Págs. 46-57] Número monográfico - Los nudges y el diseño conductual de políticas públicas

Economía del comportamiento, políticas conductuales y nudges

Luis Miller

biando en repetidas ocasiones, haciendo que la ciudadanía se enfrentara al sesgo de statu quo descrito en la tabla $2^{9}$. De igual modo, también podemos encontrar multitud de ejemplos relacionados con el coste hundido psicológico. Por ejemplo, a muchas personas les costaba volver a la normalidad después del confinamiento por lo que habían «invertido» en prepararse para el mismo con anterioridad ${ }^{10}$.

Como vemos, buena parte de los sesgos y problemas que generan las decisiones bajo riesgo parecen especialmente propicios para intervenciones informativas. En este caso es más difícil pensar en intervenciones más clásicas basadas en incentivos materiales, ya que en general el problema tiene que ver con percepciones y creencias erróneas. El mayor problema, por tanto, no sería el coste de las intervenciones, sino algo más fundamental que había apuntado al comienzo de esta sección: en la mayoría de situaciones descritas no solo tenemos un problema de riesgo, sino de incertidumbre, donde no podemos hacernos una idea más o menos clara de cuáles son las consecuencias esperadas de nuestras acciones.

En la economía del comportamiento analiza las situaciones de incertidumbre a través de la denominada «aversión a la ambigüedad», que podríamos definir como la tendencia a preferir un riesgo conocido a uno desconocido, es decir, tendemos a intentar evitar situaciones que implican ambigüedad o incertidumbre (Epstein, 1999). Sobre la aversión a la ambigüedad tenemos mucha menos evidencia que sobre la aversión al riesgo y casi toda esta evidencia viene del laboratorio. Aunque en las situaciones donde existe riesgo e incertidumbre solemos utilizar todo lo aprendido de la teoría prospectiva, en la mayoría de casos (como en la ya mencionada pandemia) la situación resulta algo más complicada, ya que no sabemos ni siquiera con qué probabilidad aproximada se va a producir un evento importante, como un contagio.

\section{POLÍTICAS CONDUCTUALES EN UN MUNDO PARCIALMENTE COOPERATIVO}

Otro de los aspectos que se le suelen escapar a las políticas tradicionales basadas en incentivos materiales es que el mundo no está poblado solo por sociópatas como la caricatura que normalmente hacemos del homo economicus. Por suerte, las políticas públicas pueden sustentarse en parte sobre las actitudes prosociales de las personas. En muchos casos, las herramientas políticas y las acciones espontáneas de las personas son más complementos que sustitutos. Pero, ¿qué son las preferencias o actitudes prosociales?, ¿dónde y cuándo es más probable encontrarlas?, y, lo que es más importante para lo que aquí nos ocupa, ¿cómo puede contribuir a la mejora de las políticas públicas?

Cuando decimos que las personas tienen preferencias sociales generalmente nos referimos a dos tipos de comportamientos que contrastan con el supuesto egoísmo del «hombre económico». Por una parte, la investigación antropológica, económica y psicológica ha llegado a la conclusión de que el tipo de preferencia o comportamiento más común en el ser humano es el de la denominada «reciprocidad fuerte» (Gintis 2009). Esta implica que los humanos tendemos a cooperar y a responder al comportamiento cooperativo de otros con nuestra propia cooperación, pero también responder al comportamiento no cooperativo de otras personas sancionándolas (informalmente o formalmente, normalmente a través de instituciones centralizadas). Esto cambia radicalmente la formulación de determinadas políticas públicas, ya que el Estado puede esperar cierto grado de cooperación y compromiso por parte de los ciudadanos. De hecho, habría muchos comportamientos imprescindibles para el funcionamiento de una sociedad (como el pago de impuestos o el respeto a ciertas normas cívicas) que serían impensables y costosísimos sin un cierto grado de cooperación voluntaria. También sería absurdo que un Estado diseñara las políticas públicas pensando en una sociedad repleta de cooperadores incondicionales. En realidad, como han mostrado multitud de estudios de laboratorio y de campo, la sociedad presenta una mezcla de «tipos» de personas que incluye tanto a cooperadores, defraudadores y a personas que están dispuestas a contribuir más o menos dependiendo del contexto. Y es trabajando sobre el contexto donde las políticas públicas pueden sacar un mayor rendimiento a la tendencia a la cooperación voluntaria.

Algunos de los nudges más conocidos intentan potenciar la cooperación voluntaria de las personas. El ejemplo de la mosca en el urinario no hace otra cosa que incitar, de forma divertida, a los usuarios a mantener los baños limpios, algo que beneficia a todo el mundo. La alternativa serían amenazas de sanciones por el incumplimiento. En general, se puede pensar en muchas intervenciones que explotan la propensión natural de los humanos a cooperar. Otro ejemplo clásico es la «teoría de las ventanas rotas» (Kelling y Co-

\footnotetext{
9 Sobre el sesgo de statu quo, ver el texto clásico de Kahneman et al. (1991).

10 Sobre el concepto de coste hundido psicológico, ver Arkes y Blumer (1985).
} 
GAPP. Nueva Época - N. 25, marzo 2021 - ISSN: 1989-8991 - DOI: https://doi.org/10.24965/gapp.i25.10866 - [Págs. 46-57] Número monográfico - Los nudges y el diseño conductual de políticas públicas

Economía del comportamiento, políticas conductuales y nudges

Luis Miller

les, 1998) que propone que manteniendo los vecindarios limpios y ordenados se conseguiría una reducción del vandalismo y la criminalidad en general.

Más allá de la tendencia natural humana a la cooperación, un segundo mecanismo relacionado con el comportamiento prosocial es nuestra adherencia a determinados principios morales interiorizados. Un ejemplo interesante de esto es que solemos ser más generosos cuando pensamos que alguien nos mira, incluso si ese alguien está solo en una fotografía. Ante la mirada de otros sentimos el compromiso (moral) a cooperar o ser generosos (Bateson et al., 2006). Otro de los ejemplos clásicos de la explotación de nuestras tendencias prosociales por los nudges es la inclusión de mensajes en cartas dirigidas a motivarnos para pagar impuestos (James, 2017) o para votar (Green y Gerber, 2015). En ambos casos no son los incentivos materiales, sino nuestra propensión a contribuir socialmente lo que se activa.

Sin embargo, el campo en el que las políticas conductuales y los nudges pueden desempeñar un mayor papel es en situaciones donde directamente un estado democrático no puede utilizar políticas coercitivas basadas en incentivos materiales. De nuevo, la pandemia de la COVID-19 nos ofrece multitud de ejemplos. Me detendré en uno que ha generado bastante discusión al respecto: las aplicaciones de teléfonos móviles dedicadas al rastreo de posibles contagios. La aplicación de rastreo es un ejemplo claro de «problema de bien público». La aplicación, como las vacunas, las mascarillas o el distanciamiento físico, es un bien público en tanto que su adopción individual beneficia al resto de la sociedad. Descargar la aplicación, como llevar mascarilla, es algo costoso desde un punto de vista individual, pero contribuye a la disminución del contagio, lo que supone una ganancia colectiva. Y es esta tensión entre los costes individuales de un comportamiento y los beneficios colectivos lo que explica que muchos bienes públicos que consideramos esenciales (reducir las emisiones contaminantes) cueste tanto alcanzarlos. Pero, ¿por qué las personas no contribuimos a los bienes públicos? Y, ¿qué pueden hacer los gobiernos para incrementar la contribución a los mismos?

La respuesta clásica es a través de sanciones. Esta sería la dinámica que ha ocurrido con las mascariIlas: al hacerlas obligatorias y sancionar su uso, acaban utilizándose de forma masiva. El problema con las aplicaciones es que no podemos obligar a que se usen. India experimentó con esa posibilidad, obligando a los trabajadores a usar la aplicación, y recibió numerosas críticas por ello ${ }^{11}$. Por tanto, nos encontramos ante el complicado problema de la contribución voluntaria a un bien público. Un bien público que, además, necesita un mínimo de contribuciones (umbral) para ser efectivo. Descartadas las sanciones, ¿qué podemos hacer? Hay tres estrategias alternativas: información, facilidad en el uso (disminuyendo el coste individual) e incentivos positivos individuales. El tratamiento informativo tiene que ver con el lanzamiento de la aplicación, para solucionar el denominado «problema de inicio»: hasta que un número suficiente de personas se unen a la tecnología. Una vez que esto ocurre, la contribución o adopción es muy rápida, hasta que llega a un punto donde se estabiliza. Por tanto, la información es esencial para solucionar el problema de inicio. Pero esto no es suficiente. Para que la aplicación no sea flor de un día es importante trabajar sobre los costes individuales de usarla, fundamentalmente a través de un diseño sencillo (reduciendo costes), y sobre los beneficios individuales de utilizarla. Este último punto es donde más están fallando los países más avanzados en el uso de las aplicaciones de rastreo. Terminaré con dos propuestas acerca de qué incentivos podrían utilizarse para que la mayoría de personas utilicen la aplicación. La primera consistiría en que todo el que se descargara/usara la aplicación entrara en una lotería para recibir un premio. Ese premio sería financiado por las multas impuestas a aquellos que se saltan comportamientos de prevención de la COVID-19. Puede parecer algo extraño, pero la ciudad de Estocolmo ha puesto en marcha una lotería muy parecida para aquellos que no se saltan los límites de velocidad ${ }^{12}$. Pero si se considera una estrategia experimental como la anterior algo muy arriesgado, podemos pensar en otra idea mucho más conservadora. Se trataría de combinar dos aplicaciones en una. Por una parte, una aplicación donde se ofrezca información acerca de la pandemia de forma simple y atractiva y, por otra, la aplicación de rastreo. Se trata de ofrecer un incentivo para el usuario de la aplicación, es decir, ofrecerle un incentivo individual, en este caso en forma de información y entretenimiento, para que adopte un comportamiento beneficioso desde el punto de vista colectivo. Como vemos, en ejemplos reales como la aplicación de rastreo, generalmente necesitamos políticas que combinen aproximaciones tradicionales basadas en incentivos e intervenciones más novedosas que aprovechen tendencias conductuales como la cooperación voluntaria que puede ser activada a través de nudges.

11 Ver este comentario de la Organización Mundial de la Salud: https://www.who.int/news-room/commentaries/detail/immunitypassports-in-the-context-of-covid-19.

12 Ver la historia aquí: https://medicalfuturist.com/swedish-speed-camera-lottery-healthy-living/. 
GAPP. Nueva Época - N.o 25, marzo 2021 - ISSN: 1989-8991 - DOI: https://doi.org/10.24965/gapp.i25.10866 - [Págs. 46-57] Número monográfico - Los nudges y el diseño conductual de políticas públicas

Economía del comportamiento, políticas conductuales y nudges

Luis Miller

\section{POLÍTICAS CONDUCTUALES E INTERACCIÓN SOCIAL}

Aunque muchas de las propuestas de políticas conductuales hacen referencia a comportamientos individuales, es difícil pensar en situaciones donde estas no se vean también influenciadas por la interacción social. Incluso en cuestiones como el ejercicio o las dietas, la influencia social, la difusión y el contagio sociales son centrales. Claro, cuando intentamos incorporar la interacción social al diseño de políticas públicas conductuales todo se complica. Pensemos en un ejemplo del que se ha hablado bastante durante la pandemia de la COVID-19: la difusión de comportamientos de prevención entre la población. Uno de los problemas que nos plantea este tipo de virus es que mientras que el contagio del mismo es muy rápido, la difusión de las medidas para combatirlo no lo es tanto. Esto se suele explicar aludiendo a la diferencia entre contagio simple y contagio complejo (Centola y Macy, 2007). El contagio del virus sería simple en tanto que solo requiere un contacto para que se produzca. Sin embargo, el contagio de las medidas más populares para prevenirlo (uso de mascarillas, distanciamiento social y medidas de higiene) es un ejemplo de contagio complejo, como suele ocurrir con la difusión de prácticas sociales, donde es necesario que estemos expuestos a múltiples contactos para que adoptemos un comportamiento. Esto conecta con la teoría del umbral (Granoveter, 1978) en el comportamiento colectivo según la cual las personas tenemos distintos umbrales para adherirnos a un comportamiento social. Hay personas que adquieren un hábito sin necesidad de que otros lo hayan hecho antes, pero hay otras que requieren que muchas otras antes lo hayan hecho.

Repasar la multitud de tipos de situaciones que caben dentro del paraguas de la interacción social es algo que va mucho más allá de los objetivos de este artículo. Tendríamos que hablar de competición, cooperación, negociación, contagio, difusión o coordinación, entre muchos otros. Mi objetivo en esta última sección es más limitado y consiste en destacar la complejidad de diseñar nudges teniendo en cuenta la interacción estratégica entre individuos. Para ello, me detendré en un tipo de interacción relativamente simple como es la coordinación. En general, los denominados problemas de coordinación representan situaciones en las que los actores tienen intereses similares y, aunque puede no importarles qué solución será la impuesta, están de acuerdo en que algún tipo de solución es necesaria (Miller, 2007). Pensemos, por ejemplo, en multitud de políticas que se han puesto en marcha en los últimos meses para reducir el número de interacciones sociales, así como los contactos físicos entre personas. En muchos casos se han utilizado diseños clásicos como separar visualmente el sentido del paso, obligándonos a andar siempre por el extremo derecho de la acera o lugar de paso. En otros se han utilizado todo tipo de señales informativas para garantizar que respetemos la distancia interpersonal de dos metros. ¿Cómo pueden contribuir las políticas conductuales y nudges a resolver problemas de coordinación?

Curiosamente, dado que los problemas de coordinación no implican conflicto de intereses, la mayoría de estos se pueden solucionar con información y, en situaciones donde las personas deban tomar decisiones más o menos instantáneas, con intervenciones conductuales o nudges. Estos deben explotar las ideas básicas detrás de la solución a cualquier problema de coordinación: la idea de prominencia (salience) y la idea de punto focal (Schelling, 1960). La prominencia hace referencia a un estado o cualidad que sobresale con respecto a otros a su alrededor. Podemos pensar en la cantidad de símbolos empleados para ordenar la circulación que utilizan colores (verde, rojo) y formas (flechas) a las que nuestro cerebro está acostumbrado. Por su parte, los puntos focales son soluciones que las personas tienden a utilizar en ausencia de comunicación porque resultan naturales, especiales o relevantes para ellas. De nuevo, estamos utilizando algunos de los mecanismos de los que hablábamos más arriba, como las opciones por defecto, para resolver en este caso un problema de interacción social. La única diferencia es que ahora no solo debemos tener en cuenta las opciones que nuestro cerebro interpreta como naturales o normales, sino aquellas que, al mismo tiempo, sabemos que también resultan naturales o normales al resto de personas con las que nos queremos coordinar explícita o implícitamente.

\section{CONCLUSIONES}

En este artículo he intentado transmitir la idea de que las políticas conductuales y los nudges están sustentadas en un sinfín de teorías y resultados empíricos procedentes de las ciencias del comportamiento que no podemos ignorar. En muchos casos estas teorías no forman parte de un conjunto coherente de principios generales, como en el caso de la economía neoclásica, sino que se han ido desarrollando para intentar explicar problemas concretos que esta teoría no llegaba a explicar satisfactoriamente, como los problemas de 
GAPP. Nueva Época - N. ${ }^{\circ}$ 25, marzo 2021 - ISSN: 1989-8991 - DOI: https://doi.org/10.24965/gapp.i25.10866 - [Págs. 46-57] Número monográfico - Los nudges y el diseño conductual de políticas públicas

Economía del comportamiento, políticas conductuales y nudges

Luis Miller

inconsistencias en el comportamiento individual a lo largo del tiempo. Pero el que no formen parte de una única gran teoría no quiere decir que no podamos describir cuáles son los fundamentos conductuales que se hayan detrás de determinadas políticas o intervenciones públicas.

En definitiva, las políticas públicas se diseñan para resolver algún tipo de problema y, por tanto, no podemos ignorara la estructura de estos problemas. Para ello en este artículo he querido definir tres tipos de situaciones dependiendo de su grado de complejidad. En primer lugar, me he centrado en problemas de decisión individual, los cuales han recibido un trato privilegiado dentro de la literatura sobre nudge. En concreto he repasado problemas y teorías relacionados con las decisiones intertemporales y las decisiones bajo riesgo e incertidumbre. Dada la relevancia de este tipo de problemas en la literatura conductual, tenemos muchos ejemplos de cómo aplicar políticas conductuales y cómo estas interaccionan con políticas más clásicas basadas en incentivos.

En segundo lugar, he repasado situaciones donde los humanos tenemos en cuenta a otras personas a la hora de decidir sobre nuestro comportamiento. El ejemplo utilizado ha sido el de las preferencias y actitudes prosociales, por ejemplo, en contextos de donaciones altruistas o de cooperación voluntaria. Aquí el problema es, de nuevo, cómo el Estado puede aprovechar algunas tendencias individuales en este sentido para no tener que fiarlo todo a políticas de incentivos y sanciones. Un caso especialmente relevante son las situaciones donde la cooperación es necesaria pero el Estado no puede imponerla por cuestiones éticas, como es el caso de las aplicaciones para teléfonos móviles de rastreo de contactos para hacer un seguimiento de los posibles contagios de una enfermedad. Este último caso supone un escenario privilegiado para las políticas conductuales, ya que son las únicas posibles.

Por último, he hecho una pequeña incursión en el tipo de situación más compleja para el diseño, puesta en marcha y seguimiento de políticas conductuales: las situaciones de interacción social o estratégica. En este tipo de situaciones ya no solo se trata de cambiar el comportamiento individual en una determinada dirección, sino que este debe coordinarse con el de otras personas. La idea de punto focal como solución a los problemas de coordinación es de especial relevancia para las políticas conductuales, aunque aún tenemos relativamente poca investigación que nos permita imaginar políticas y nudges al respecto.

Como en tantos ámbitos de las políticas públicas, la retroalimentación entre investigación básica, políticas públicas y evaluación de estas es fundamental. El campo de las políticas conductuales es relativamente reciente y todavía hay áreas donde las teorías y resultados empíricos son escasos. Sin embargo, no podemos caer en el error de separar la caja de herramientas conductual de las teorías y datos que han ayudado a perfilarla. De lo contrario, usadas de forma irreflexiva como la última moda en intervenciones, las políticas conductuales podrían perder buena parte de su efectividad.

\section{REFERENCIAS BIBLIOGRÁFICAS}

Ariely, D. y Zakay, D. (2001). A timely account of the role of duration in decision making. Acta Psychologica, 108(2), 187-207. https://doi.org/10.1016/s0001-6918(01)00034-8

Arkes, H. y Blumer, C. (1985). The psychology of sunk cost. Organizational Behavior and Human Decision Processes, 35(1), 124-140. https://doi.org/10.1016/0749-5978(85)90049-4

Bateson, M., Nettle, D. y Roberts, G. (2006). Cues of being watched enhance cooperation in a real-world setting. Biology letters, 2(3), 412-414. https://doi.org/10.1098/rsbl.2006.0509

Brandstätter, H. y Güth, W. (1994). Essays on economic psychology. Springer.

Camerer, C. (2000). Prospect theory in the wild: evidence from the field. En Choices, values and frames, Editado por Daniel Kahneman y Amos Tversky. Cambridge university press.

Centola, D. y Macy, M. (2007). Complex contagions and the weakness of long ties. American Journal of Sociology, 113(3), 702-734. https://doi.org/10.1086/521848

Charness, G. y Gneezy, U. (2009). Incentives to exercise. Econometrica, 77(3), 909-931. https://www.jstor.org/ stable/40263846

Epstein, L. (1999). A definition of uncertainty aversion. The Review of Economic Studies, 66(3), 579-608. https://doi. org/10.1111/1467-937X.00099

Forberger, S., Reisch, L., Kampfmann, T. y Zeeb, H. (2019). Nudging to move: a scoping review of the use of choice architecture interventions to promote physical activity in the general population. International Journal of Behavioral Nutrition and Physical activity, 16, artículo 77. https://ijbnpa.biomedcentral.com/articles/10.1186/s12966-0190844-z

Frederick, S., Loewenstein, G. y O'Donoghue, T. (2002). Time discounting and time preference: a critical review. Journal of Economic Literature, 40(2), 351-401. https://doi.org/10.1257/002205102320161311 
GAPP. Nueva Época - N. ${ }^{\circ}$ 25, marzo 2021 - ISSN: 1989-8991 - DOI: https://doi.org/10.24965/gapp.i25.10866 - [Págs. 46-57] Número monográfico - Los nudges y el diseño conductual de políticas públicas

Economía del comportamiento, políticas conductuales y nudges

Luis Miller

Galla, B. y Duckworth, A. (2015). More than resisting temptation: beneficial habits mediate the relationship between self-control and positive life outcomes. Journal of Personality and Social Psychology, 109(3), 508-525. https://doi. org/10.1037/pspp0000026

Green, D. y Gerber, A. (2015). Get out the vote: how to increase voter turnout. Brookings Institution Press. https://www. jstor.org/stable/10.7864/j.ctt1657t5x

Gilovich, T., Griffin, D. y Kahneman, D. (2002). Heuristics and biases: the psychology of intuitive judgment. Cambridge University Press.

Gintis, H. (2009). The bounds of reason. game theory and the unification of behavioral sciences. Princeton University Press.

Gigerenzer, G. y Selten, R. (2002). Bounded rationality: the adaptive toolbox. MIT Press.

Gneezy, U., Meier, S. y Rey-Biel, P. (2011). When and why incentives (don't) work to modify behavior. Journal of Economic Perspectives, 25(4), 191-210. https://doi.org/10.1257/jep.25.4.191

Granovetter, M. (1978). Threshold models of collective behavior. American Journal of Sociology, 83(6), 1.420-1.443. http://www.jstor.org/stable/2778111

Hertwig, R., Pachur, T. y Kurzenhäuser, S. (2005). Judgments of Risk Frequencies: Tests of Possible Cognitive Mechanisms. Journal of Experimental Psychology: Learning, Memory, and Cognition, 31(4), 621-642. https://doi. org/10.1037/0278-7393.31.4.621

Hossain, T. y List, J. (2012). The behavioralist visits the factory: increasing productivity using simple framing manipulations. Management Science, 58(12), 2.151-2.167. https://doi.org/10.1287/mnsc.1120.1544

James, M. (2017). Handbook of behavioral economics and smart decision-making: rational decision-making within the bounds of reason. Edward Elgar Publishing.

Johnson, E. y Golstein, D. (2003). Do defaults save lives? Science, 302(5.649), 1.338-1.339. https://doi.org/10.1126/ science. 1091721

Kahneman, D., Knetsch, J. y Thaler, R. (1991). Anomalies: The Endowment Effect, Loss Aversion, and Status Quo Bias. Journal of Economic Perspectives, 5(1), 193-206. https://doi.org/10.1257/jep.5.1.193

Kahneman, D. (2012). Pensar rápido, pensar despacio. Debate.

Kelling, C. y Coles, G. (1998). Fixing broken windows: restoring order and reducing crime in our communities. Simon \& Schuster.

Knight, F. (1921). Risk, uncertainty and profit. Houghton Mifflin Harcourt.

Kucharski, A. (2020). Las reglas del contagio. Capitán Swing.

Laibson, D. (1997). Golden eggs and hyperbolic discounting. Quarterly Journal of Economics, 112(2), 443-477. https:// doi.org/10.1162/003355397555253

Miller, L. (2007). Coordinación y acción colectiva. Revista Internacional de Sociología, 65(46), 161-183. https://doi. org/10.3989/ris.2007.i46.8

O'Donoghue, T. y Rabin, M. (2015). Present bias: lessons learned and to be learned. American Economic Review, 105(5), 273-279. https://doi.org/10.1257/aer.p20151085

Schelling, T. (1960). The strategy of conflict. Harvard University Press.

Simon, H. (1955). A behavioral model of rational choice. The Quarterly Journal of Economics, 69(1), 99-118. https://doi. org/10.2307/1884852

Stigler, G. y Becker, G. (1977). De Gustibus Non Est Disputandum. The American Economic Review, 67(2), 76-90. https://www.jstor.org/stable/1807222

Thaler, R. y Sustein, C. (2008). Nudge: improving decisions about health, wealth, and happiness. Yale University Press.

Tversky, A. y Kahneman, D. (1981). The framing of decisions and the psychology of choice. Science, 211(4.481), 453458. https://doi.org/10.1126/science. 7455683

Tversky, A. y Kahneman, D. (1974). Judgment under uncertainty: heuristics and biases. Science, 185(4.157), 1.1241.131. https://doi.org/10.1126/science. 185.4157 .1124 